\title{
In memoriam Peter Braun (1927-2019)
}

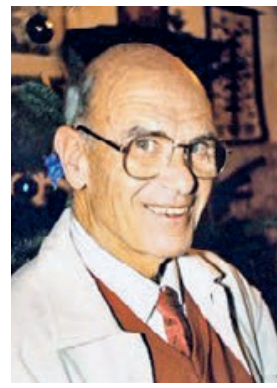

Peter Braun
Dr. med. Thomas Kehl, MBA Facharzt Orthopädische Chirurgie und Traumatologie Klinikstrasse 4 CH-7272 Davos Clavadel thomaskehl[at]bluewin.ch
Peter wurde am 21. Dezember 1927 in Zürich geboren und ist in Zollikon aufgewachsen. Seine Eltern waren Dr. Friedrich Braun, Chefarzt der Schweizerischen Anstalt für Epilepsie in Zürich, und Frau Ida Hofer-Braun.

\section{Chefarzt mit 33 Jahren}

Nach dem Staatsexamen in Zürich und der medizinischen Weiterbildung an der Schweizerischen Anstalt für Epilepsie in Zürich, am Pathologischen Institut der Universität Zürich, an der medizinischen Abteilung des Stadtspitals Waid und an der medizinischen Klinik des Universitätsspitals Zürich wurde Peter Braun am 1. Januar 1961 mit erst 33 Jahren zum Chefarzt der Zürcher Hochgebirgsklinik Davos Clavadel gewählt. Weil er das medizinische Wissen, vor allem aber auch aussergewöhnliche Führungsqualitäten und grosses menschliches Einfühlungsvermögen hatte, konnte er das einstige Lungensanatorium bis zu seiner Pensionierung 1992 erfolgreich in eine mehrdisziplinäre Rehabilitationsklinik umwandeln.

\section{Gründer des «Internationalen Diagnostik Kurses Davos» und des Medizinmuseums}

Zusammen mit Alois Rüttimann gründete Peter Braun 1969 den heute bestens bekannten Internationalen Diagnostik Kurs Davos (IDKD). Dies stellt einen Meilenstein in der beruflichen Karriere von Peter Braun dar. Er

\section{Der Kongress wurde zu einem der weltweit angesehensten Fortbildungskurse in medizini- scher Radiologie.}

schuf in Davos eine einzigartige Ausbildungsstätte in medizinischer Radiologie. Die Idee, mit den erfahrensten und vorwiegend angelsächsischen Radiologieexperten alljährlich den Kurs zu organisieren, führte dazu, dass der Kongress zu einem der weltweit angesehensten und führenden Fortbildungskurse in medizinischer Radiologie wurde und heute neben Davos auch in Griechenland, Asien und Brasilien durchgeführt wird.

Peter Braun gründete nach seiner Pensionierung das Medizinmuseum in Davos. In diesem Museum können die Besucherinnen und Besucher interessante Details über die Meilensteine der Geschichte der Tuberkulose entdecken und medizinische Geräte und Instrumente aus den ersten Davoser Sanatorien bestaunen. Die medizinhistorische Sammlung umfasst mehr als 500 medizinische Geräte, Instrumente und Dokumente aus den Sanatorien und Kliniken.

\section{Expedition zum Dhaulagiri}

Neben seiner medizinischen Laufbahn war Peter Braun zeitlebens ein begeisterter Bergsteiger. Der absolute Höhepunkt und eines der bedeutendsten Erlebnisse im Leben von Peter Braun war der Versuch der Erstbesteigung des Dhaulagiri, des siebthöchsten Bergs

\section{Am 29. Mai 1953 brach er zusammen mit}

Rudi Schatz zum Angriff auf den Gipfel auf.

dieser Welt. Am 15. März 1953 startete er die Expedition zusammen mit der Schweizerischen Himalayaexpedition des Akademischen Alpenclubs Zürich. Am 29. Mai brach er zusammen mit Rudi Schatz vom Lager 5 zum Angriff auf den Gipfel auf. Auf etwas über 7700 Meter Höhe gab es aber kein Weiterkommen mehr. Das Risiko für einen weiteren Aufstieg in diesem technisch äusserst anspruchsvollen Gelände war viel zu gross, so dass sie die Umkehr beschlossen. Erwähnenswert ist, dass erst 28 Jahre später Japaner auf dieser Route den Gipfel des Dhaulagiri auf 8172 Metern erreichten.

Peter Braun war seit 1969 aktives Mitglied des Rotary Clubs Davos Klosters. Er hat die Idee und das Gedankengut von Rotary beispielhaft im Alltag gelebt und gefördert.

\section{«Papa Braun»}

Peter Braun schaffte es, wie kein Zweiter, Familie, Beruf, Sport und Freizeit unter einem Dach zu vereinen. Seine Menschlichkeit, fachlichen Qualitäten und Schaffenskraft machten ihn zu einem grossartigen Menschen und Arzt. Seine Beliebtheit bei seinen Mitarbeitenden in der Zürcher Hochgebirgsklinik Clavadel zeigte sich unter anderem darin, dass er in seinem Team oft «Papa Braun» genannt wurde.

Alle, die ihn kannten, werden Peter Braun vermissen und ihm ein ehrendes Andenken bewahren.

Thomas Kehl 